\title{
EXACT NEUMANN BOUNDARY CONTROLLABILITY FOR PROBLEMS OF TRANSMISSION OF THE WAVE EQUATION
}

\author{
WEIJIU LIU and GRAHAM H. WILLIAMS \\ Department of Mathematics, University of Wollongong, Northfields Avenue, Wollongong, \\ NSW 2522, Australia \\ e-mail:w.liu@uow.edu.au;ghw@uow.edu.au
}

(Received 10 April, 1997)

\begin{abstract}
Using the Hilbert Uniqueness Method, we study the problem of exact controllability in Neumann boundary conditions for problems of transmission of the wave equation. We prove that this system is exactly controllable for all initial states in $L^{2}(\Omega) \times\left(H^{1}(\Omega)\right)^{\prime}$.
\end{abstract}

1. Introduction. Throughout this paper, let $\Omega$ be a bounded domain (open, connected, and nonempty) in $\mathrm{R}^{n}(n \geq 1)$ with a boundary $\Gamma=\partial \Omega$ of class $C^{2}$, and $\Omega_{1}$ given with $\bar{\Omega}_{1} \subset \Omega$ and $\Gamma_{1}=\partial \Omega_{1}$ of class $C^{2}$. Let $T>0$. Set $\Omega_{2}=\Omega-\Omega_{1}, Q=\Omega \times(0, T)$, $Q_{1}=\Omega_{1} \times(0, T), Q_{2}=\Omega_{2} \times(0, T), \Sigma=\Gamma \times(0, T), \Sigma_{1}=\Gamma_{1} \times(0, T)$.

In [6], Lions studied the problem of exact controllability with Dirichlet boundary conditions for problems of transmission of the wave equation by introducing the Hilbert Uniqueness Method (HUM for short). Later, Nicaise [10-12] further considered this problem in $\mathrm{R}^{2}$ with singularities.

In this paper, we consider the following Neumann boundary controllability problem in $\mathrm{R}^{n}$ : For suitable times $T>0$ and every initial condition $\left\{y^{0}, y^{1}\right\}$, does there exist a control function $g$ such that the solution $y=y(x, t ; g)$ of the Neumann boundary value problem

$$
\left\{\begin{array}{cc}
y^{\prime \prime}-A(x) \Delta y=0 & \text { in } Q, \\
y(x, 0)=y^{0}(x), y^{\prime}(x, 0)=y^{1}(x) & \text { in } \Omega, \\
\frac{\partial y_{2}}{\partial v}=g & \text { on } \Sigma, \\
y_{1}=y_{2}, \quad a_{1}=\frac{\partial y_{1}}{\partial v}=a_{2} \frac{\partial y_{2}}{\partial v} & \text { on } \Sigma_{1}
\end{array}\right.
$$

satisfies

$$
y(x, T ; g)=y^{\prime}(x, T ; g)=0 \quad \text { in } \Omega ?
$$

In (1.1), $y_{1}=\left.y\right|_{\Omega_{1}}, y_{2}=\left.y\right|_{\Omega_{2}}, v$ is the unit normal of $\Gamma$ or $\Gamma_{1}$ pointing towards the exterior of $\Omega$ or $\Omega_{1}$, and $A(x)$ is given by

$$
A(x)= \begin{cases}a_{1}, & x \in \Omega_{1}, \\ a_{2}, & x \in \Omega_{2},\end{cases}
$$

where $a_{1}$, and $a_{2}$ are positive constants.

We will prove that if $\Omega_{1}$ is star-shaped and $a_{2} \leq a_{1}$, then for all initial states

$$
\left\{y^{0}, y^{1}\right\} \in L^{2}(\Omega) \times\left(H^{1}(\Omega)\right)^{\prime},
$$


there exists a control function $g$ such that the solution $y=y(x, t ; g)$ of $(1.1)$ satisfies (1.2). Here and in the sequel, $H^{\mathrm{s}}(\Omega)$ always denotes the usual Sobolev space for $s \in R$.

The plan for the rest of this paper is as follows. In Section 2, we present the theorem about the existence and uniqueness of solutions of the problem of transmission. The estimates for the solutions (i.e., the so-called "inverse inequality") are given in Section 3. The main theorems of this paper are established in Section 4.

2. Homogeneous boundary problems. Consider the following homogeneous boundary problem

$$
\left\{\begin{array}{cc}
u^{\prime \prime}-A(x) \Delta u=f & \text { in } Q, \\
u(x, 0)=u^{0}(x), u^{\prime}(x, 0)=u^{1}(x) & \text { in } \Omega \\
\frac{\partial u_{2}}{\partial v}=0 & \text { on } \Sigma \\
u_{1}=u_{2}, a_{1}=\frac{\partial u_{1}}{\partial v}=a_{2} \frac{\partial u_{2}}{\partial v} & \text { on } \Sigma_{1}
\end{array}\right.
$$

where $u_{1}=\left.u\right|_{\Omega_{1}}$ and $u_{2}=\left.u\right|_{\Omega_{2}}$. Set

$$
\begin{array}{r}
H^{2}\left(\Omega_{1}, \Omega_{2}\right)=\left\{u: u \in H^{1}(\Omega) ; u_{i}=\left.u\right|_{\Omega_{i}} \in H^{2}\left(\Omega_{i}\right), i=1,2 ;\right. \\
\left.a_{1} \frac{\partial u_{1}}{\partial v}=a_{2} \frac{\partial u_{2}}{\partial v} \text { on } \Gamma_{1} ; \frac{\partial u_{2}}{\partial v}=0 \text { on } \Gamma\right\}
\end{array}
$$

with the norm

$$
\|u\|_{H^{2}\left(\Omega_{1}, \Omega_{2}\right)}=\left[\|u\|_{H^{1}(\Omega)}^{2}+\left\|\Delta u_{1}\right\|_{L^{2}\left(\Omega_{1}\right)}^{2}+\left\|\Delta u_{2}\right\|_{L^{2}\left(\Omega_{2}\right)}^{2}\right]^{1 / 2}
$$

The well-posedness of (2.1) is by now well known ([3], Vol.5, Chap. XVIII] and [4]). We have the following result.

THEOREM 2.1. (i) Suppose $\Gamma$ and $\Gamma_{1}$ are Lipschitz. Then, for any initial condition $\left(u^{0}, u^{1}\right) \in H^{1}(\Omega) \times L^{2}(\Omega)$ and $f \in L^{1}\left(0, T ; L^{2}(\Omega)\right)$, problem (2.1) has a unique weak solution $u$ with

$$
u \in C\left([0, T] ; H^{1}(\Omega)\right) \cap C^{1}\left([0, T] ; L^{2}(\Omega)\right)
$$

Moreover, there exists a constant $C>0$ such that for every $t \in[0, T]$

$$
\|u(t)\|_{H^{1}(\Omega)}+\left\|u^{\prime}(t)\right\|_{L^{2}(\Omega)} \leq C\left[\left\|u^{0}\right\|_{H^{1}(\Omega)}+\left\|u^{1}\right\|_{L^{2}(\Omega)}+\|f\|_{L^{1}\left(0, T ; L^{2}(\Omega)\right.}\right] .
$$

(ii) Suppose $\Gamma$ and $\Gamma_{1}$ are of class $C^{2}$. Then for any initial condition $\left(u^{0}, u^{1}\right) \in H^{2}\left(\Omega_{1}, \Omega_{2}\right) \times H^{1}(\Omega)$ and $f \in L^{1}\left(0, T ; H^{1}(\Omega)\right)$, problem (2.1) has a unique strong solution $u$ with 


$$
u \in C\left([0, T] ; H^{2}\left(\Omega_{1}, \Omega_{2}\right)\right) \cap C^{1}\left([0, T] ; H^{1}(\Omega)\right) .
$$

Moreover, there exists a constant $C>0$ such that for every $t \in[0, T]$

$$
\begin{aligned}
& \left\|u^{\prime}(t)\right\|_{H^{1}(\Omega)}+\|u(t)\|_{H^{2}\left(\Omega_{1}, \Omega_{2}\right)} \\
& \leq C\left[\left\|u^{1}\right\|_{H^{1}(\Omega)}+\left\|u^{0}\right\|_{H^{2}\left(\Omega_{1}, \Omega_{2}\right)}+\|f\|_{L^{1}\left(0, T ; H^{1}(\Omega)\right)}\right] .
\end{aligned}
$$

3. Basic inequalities. We adopt the notation used in $[6,7]$ as follows. Let $x^{0} \in R^{n}$, and set

$$
\begin{gathered}
m(x)=x-x^{0}=\left(x_{k}-x_{k}^{0}\right) . \\
\Gamma\left(x^{0}\right)=\left\{x \in \Gamma: m(x) \cdot v(x)=m_{k}(x) \cdot v_{k}(x)>0\right\} \\
\Gamma_{*}\left(x^{0}\right)=\Gamma-\Gamma\left(x^{0}\right)=\{x \in \Gamma: m(x) \cdot v(x) \leq 0\} \\
\Sigma\left(x^{0}\right)=\Gamma\left(x^{0}\right) \times(0, T) \\
\Sigma_{*}\left(x^{0}\right)=\Gamma_{*}\left(x^{0}\right) \times(0, T) \\
R\left(x^{0}\right)=\max _{x \in \bar{\Omega}}|m(x)|=\max _{x \in \bar{\Omega}}\left|\Sigma_{k=1}^{n}\left(x_{k}-x_{k}^{0}\right)^{2}\right|^{\frac{1}{2}} .
\end{gathered}
$$

where $v$ denotes the outward unit normal to $\Gamma$.

We define the energy of the solution $u$ of (2.1) by

$$
E(t)=\frac{1}{2} \int_{\Omega}\left[\left|u^{\prime}(x, t)\right|^{2}+A(x)|\nabla u|^{2}\right] d x,
$$

If $f=0$, then we have the classical result (see $[\mathbf{6}, 9])$

$$
E(t) \equiv E(0) .
$$

The following identities are essential for establishing the follow-up inverse inequalities.

Lemma 3.1. Let $q=\left(q_{k}\right)$ a vector field in $\left[C^{1}(\bar{\Omega})\right]^{n}$. Suppose $u$ is the strong solution of (2.1) in the sense of (ii) of Theorem 2.1. Then the following identity holds: 


$$
\begin{aligned}
& \frac{1}{2} \int_{\Sigma} q_{k} v_{k}\left(\left|u_{2}^{\prime}\right|^{2}-a_{2}\left|\nabla_{\sigma} u_{2}\right|^{2}\right) d \Sigma \\
& \left.=\int_{Q}(t), q_{k} \frac{\partial u(t)}{\partial x_{k}}\right)\left.\right|_{0} ^{T}+\int_{Q} A(x) \frac{\partial u}{\partial x_{j}} \frac{\partial q_{k}}{\partial x_{i}} \frac{\partial u}{\partial x_{k}} d x d t \\
& +\frac{1}{2} \int_{Q} \frac{\partial q_{k}}{\partial x_{k}}\left(\left|u^{\prime}\right|^{2}-A(x)|\nabla u|^{2}\right) d x d t \\
& -a_{1}\left(1-\frac{a_{1}}{a_{2}}\right) \int_{\Sigma_{1}} q_{k} v_{k}\left|\frac{\partial u_{1}}{\partial v}\right|^{2} d \Sigma \\
& -\frac{1}{2} \int_{\Sigma_{1}} q_{k} v_{k}\left(a_{2}\left|\nabla u_{2}\right|^{2}-a_{1}\left|\nabla u_{1}\right|^{2}\right) d \Sigma-\int_{Q} q_{k} \frac{\partial u}{\partial x_{k}} f d x d t,
\end{aligned}
$$

where

$$
\left(u^{\prime}(t), q_{k} \frac{\partial u(t)}{\partial x_{k}}\right)=\int_{\Omega} u^{\prime}(t) q_{k} \frac{\partial u(t)}{\partial x_{k}} d x
$$

and $\nabla_{\sigma} u=\left\{\sigma_{j} u\right\}_{j=1}^{n}$ denotes the tangential gradient of $u$ on $\Gamma$. (See [6, p.137].)

REMARK 3.2. If $n=1$, then (3.1) becomes

$$
\begin{aligned}
& \frac{1}{2} \int_{\Sigma} q v\left|u_{2}^{\prime}\right|^{2} d \Sigma \\
& =\left.\left(u^{\prime}(t), q_{k} \frac{\partial u(t)}{\partial x}\right)\right|_{0} ^{T}+\int_{Q} A(x)\left|\frac{\partial u}{\partial x}\right|^{2} \frac{\partial q}{\partial x} d x d t \\
& +\frac{1}{2} \int_{Q} \frac{\partial q}{\partial x}\left(\left|u^{\prime}\right|^{2}-A(x)\left|\frac{\partial u}{\partial x}\right|^{2}\right) d x d t-a_{1}\left(1-\frac{a_{1}}{a_{2}}\right) \int_{\Sigma_{1}} q v\left|\frac{\partial u_{1}}{\partial v}\right|^{2} d \Sigma \\
& -\frac{1}{2} \int_{\Sigma_{1}} q v\left(a_{2}\left|\nabla u_{2}\right|^{2}-a_{1}\left|\nabla u_{1}\right|^{2}\right) d \Sigma-\int_{Q} q \frac{\partial u}{\partial x} f d x d t .
\end{aligned}
$$

This is a generalisation of the identity in Remark 1.5 of [6].

Proof. Multiplying (2.1) by $q_{k} \frac{\partial u}{\partial x_{k}}$ and integrating on $Q$, we have

$$
\int_{Q} q_{k} \frac{\partial u}{\partial x_{k}} u^{\prime \prime} d x d t-\int_{Q} q_{k} \frac{\partial u}{\partial x_{k}} A(x) \Delta u d x d t=\int_{Q} q_{k} \frac{\partial u}{\partial x_{k}} f d x d t .
$$

Integrating by parts, we obtain 


$$
\begin{aligned}
& \int_{Q} q_{k} \frac{\partial u}{\partial_{k}} u^{\prime \prime} d x d t \\
& =\left.\left(u^{\prime}(t), q_{k} \frac{\partial u(t)}{\partial x_{k}}\right)\right|_{0} ^{T}-\frac{1}{2} \int_{\Sigma_{1}} q_{k} v_{k}\left|u_{1}^{\prime}\right|^{2} d \Sigma+\frac{1}{2} \int_{Q_{1}} \frac{\partial q_{k}}{\partial x_{k}}\left|u_{1}^{\prime}\right|^{2} d x d t \\
& +\frac{1}{2} \int_{\Sigma_{1}} q_{k} v_{k}\left|u_{2}^{\prime}\right|^{2} d \Sigma-\frac{1}{2} \int_{\Sigma} q_{k} v_{k}\left|u_{2}^{\prime}\right|^{2} d \Sigma+\frac{1}{2} \int_{Q_{2}} \frac{\partial q_{k}}{\partial x_{k}}\left|u_{2}^{\prime}\right|^{2} d x d t \\
& =\left.\left(u^{\prime}(t), q_{k} \frac{\partial u(t)}{\partial x_{k}}\right)\right|_{0} ^{T}-\frac{1}{2} \int_{\Sigma} q_{k} v_{k}\left|u_{2}^{\prime}\right|^{2} d \Sigma+\frac{1}{2} \int_{Q} \frac{\partial q_{k}}{\partial x_{k}}\left|u^{\prime}\right|^{2} d x d t,
\end{aligned}
$$

and

$$
\begin{aligned}
& \int_{Q} A(x) q_{k} \frac{\partial u}{\partial x_{k}} \Delta u d x d t \\
& =\int_{\Sigma_{1}} a_{1} \frac{\partial u_{1}}{\partial v} q_{k} \frac{\partial u_{1}}{\partial x_{k}} d \Sigma-\int_{Q_{1}} a_{1} \frac{\partial u_{1}}{\partial x_{j}} \frac{\partial}{\partial x_{j}}\left(q_{k} \frac{\partial u_{1}}{\partial x_{k}}\right) d x d t \\
& -\int_{\Sigma_{1}} a_{2} \frac{\partial u_{2}}{\partial v} q_{k} \frac{\partial u_{2}}{\partial x_{k}} d \Sigma-\int_{Q_{2}} a_{2} \frac{\partial u_{2}}{\partial x_{j}} \frac{\partial}{\partial x_{j}}\left(q_{k} \frac{\partial u_{2}}{\partial x_{k}}\right) d x d t .
\end{aligned}
$$

But,

$$
\begin{aligned}
& \int_{Q_{1}} a_{1} q_{k} \frac{\partial u_{1}}{\partial x_{j}} \frac{\partial^{2} u_{1}}{\partial x_{k} \partial x_{j}} d x d t \\
& =\frac{1}{2} \int_{Q_{1}} a_{1} q_{k} \frac{\partial}{\partial x_{k}}\left|\nabla u_{1}\right|^{2} d x d t \\
& =\frac{1}{2} \int_{\Sigma_{1}} a_{1} q_{k} v_{k}\left|\nabla u_{1}\right|^{2} d \Sigma-\frac{1}{2} \int_{Q_{1}} a_{1}\left|\nabla u_{1}\right|^{2} \frac{\partial q_{k}}{\partial x_{k}} d x d t
\end{aligned}
$$

and

$$
\begin{aligned}
& \int_{Q_{2}} a_{2} q_{k} \frac{\partial u_{2}}{\partial x_{j}} \frac{\partial^{2} u_{2}}{\partial x_{k} \partial x_{i}} d x d t \\
& =\frac{1}{2} \int_{Q_{2}} a_{2} q_{k} \frac{\partial}{\partial x_{k}}\left|\nabla u_{2}\right|^{2} d x d t \\
& =-\frac{1}{2} \int_{\Sigma_{1}} a_{2} q_{k} v_{k}\left|\nabla u_{2}\right|^{2} d \Sigma+\frac{1}{2} \int_{\Sigma} a_{2} q_{k} v_{k}\left|\nabla u_{2}\right|^{2} d \Sigma \\
& -\frac{1}{2} \int_{Q_{1}} a_{2}\left|\nabla u_{2}\right|^{2} \frac{\partial q_{k}}{\partial x_{k}} d x d t
\end{aligned}
$$

Noting that $\left|\nabla u_{2}\right|^{2}=\left|\nabla_{\sigma} u_{2}\right|^{2}$ on $\Sigma$, it follows from (3.4), (3.5), and (3.6) that 


$$
\begin{aligned}
& \int_{Q} A(x) q_{k} \frac{\partial u}{\partial x_{k}} \Delta u d x d t \\
& =\int_{\Sigma_{1}} a_{1} \frac{\partial u_{1}}{\partial v} q_{k}\left(\frac{\partial u_{1}}{\partial x_{k}}-\frac{\partial u_{2}}{\partial x_{k}}\right) d \Sigma-\int_{Q} A(x) \frac{\partial u}{\partial x_{j}} \frac{\partial q_{k}}{\partial x_{j}} \frac{\partial u}{\partial x_{k}} d x d t \\
& +\frac{1}{2} \int_{\Sigma_{1}} q_{k} v_{k}\left(a_{2}\left|\nabla u_{2}\right|^{2}-a_{1}\left|\nabla u_{1}\right|^{2}\right) d \Sigma \\
& -\frac{1}{2} \int_{\Sigma} a_{2} q_{k} v_{k}\left|\nabla_{\sigma} u_{2}\right|^{2} d \Sigma+\frac{1}{2} \int_{Q} A(x)|\nabla u|^{2} \frac{\partial q_{k}}{\partial x_{k}} d x d t .
\end{aligned}
$$

Since

$$
a_{1} \frac{\partial u_{1}}{\partial v}=a_{2} \frac{\partial u_{2}}{\partial v} \text { and } \sigma_{k} u_{1}=\sigma_{k} u_{2} \text { on } \Sigma_{1}
$$

and

$$
\frac{\partial u_{1}}{\partial x_{k}}=v_{k} \frac{\partial u_{1}}{\partial v}+\sigma_{k} u_{1}, \quad \frac{\partial u_{2}}{\partial x_{k}}=v_{k} \frac{\partial u_{2}}{\partial v}+\sigma_{k} u_{2}
$$

it follows from (3.2), (3.3), and (3.7) that

$$
\begin{aligned}
& \int_{Q} f q_{k} \frac{\partial u}{\partial x_{k}} d x d t \\
& =\left.\left(u^{\prime}(t), q_{k} \frac{\partial u(t)}{\partial x_{k}}\right)\right|_{0} ^{T}-\frac{1}{2} \int_{\Sigma} q_{k} v_{k}\left|u_{2}^{\prime}\right|^{2} d \Sigma+\frac{1}{2} \int_{Q} \frac{\partial q_{k}}{\partial x_{k}}\left|u^{\prime}\right|^{2} d x d t \\
& -\int_{\Sigma_{1}} a_{1}\left(1-\frac{a_{1}}{a_{2}}\right) q_{k} v_{k}\left|\frac{\partial u_{1}}{\partial v}\right|^{2} d \Sigma+\int_{Q} A(x) \frac{\partial u}{\partial x_{j}} \frac{\partial q_{k}}{\partial x_{j}} \frac{\partial u}{\partial x_{k}} d x d t \\
& -\frac{1}{2} \int_{\Sigma_{1}} q_{k} v_{k}\left(a_{2}\left|\nabla u_{2}\right|^{2}-a_{1}\left|\nabla u_{1}\right|^{2}\right)+\frac{1}{2} \int_{\Sigma} a_{2} q_{k} v_{k}\left|\nabla_{\sigma} u_{2}\right|^{2} d \Sigma \\
& -\frac{1}{2} \int_{Q} A(x)|\nabla u|^{2} \frac{\partial q_{k}}{\partial x_{k}} d x d t .
\end{aligned}
$$

This is (3.1).

Lemma 3.3. Suppose there exists $x^{0} \in \Omega_{1}$ such that $m(x) \cdot v(x) \geq 0$ on $\Gamma_{1}$ where $v$ is directed towards the exterior of $\Omega_{1}$. Assume $a_{2} \leq a_{1}$ and $T>\frac{2 R\left(x^{0}\right)}{\sqrt{a_{2}}}$. Then for all weak solutions $u$ of (2.1) with initial conditions $\left(u^{0}, u^{1}\right) \in H^{1}(\Omega) \times L^{2}(\Omega)$ and $f=0$, there exists $C(T)>0$ such that 
EXACT NEUMANN BOUNDARY CONTROLLABILITY

$$
\begin{aligned}
& \int_{\Sigma} m_{k} v_{k}\left(\left|u_{2}^{\prime}\right|^{2}-a_{2}\left|\nabla_{\sigma} u_{2}\right|^{2}\right) d \Sigma+\int_{\Gamma} m_{k} v_{k}\left(\left|u_{2}(0)\right|^{2}+\left|u_{2}(T)\right|^{2}\right) d \Gamma \\
& \geq C(T)\left(\left\|u^{0}\right\|_{H^{1}(\Omega)}^{2}+\left\|u^{1}\right\|_{L^{2}(\Omega)}^{2}\right) .
\end{aligned}
$$

In the case $n=1$, the term $a_{2}\left|\nabla_{\sigma} \mathrm{u}_{2}\right|^{2}$ on the left-hand side of (3.8) disappears.

REMARK 3.4. If $\Omega_{1}$ is star-shaped (see [14], p.294), then the condition on $\Omega_{1}$ in the lemma is fulfilled.

Proof. We prove the lemma only in the case of $n>1$. We omit the proof in the case of $n=1$ because it is just a combination of the following proof with Lemma 1.4 of ([6], chap. 3, p. 142).

Taking $q_{k}=m_{k}$ in Lemma 3.1, we have

$$
\begin{aligned}
& \frac{1}{2} \int_{\Sigma} m_{k} v_{k}\left(\left|u_{2}^{\prime}\right|^{2}-a_{2}\left|\nabla_{\sigma} u_{2}\right|^{2}\right) d \Sigma \\
& \left.=u^{\prime}(t), m_{k} \frac{\partial u(t)}{\partial x_{k}}\right)\left.\right|_{0} ^{T}+\int_{Q} A(x)|\nabla u|^{2} d x d t \\
& +\frac{n}{2} \int_{Q}\left(\left|u^{\prime}\right|^{2}-A(x)|\nabla u|^{2}\right) d x d t-a_{1}\left(1-\frac{a_{1}}{a_{2}}\right) \int_{\Sigma_{1}} m_{k} v_{k}\left|\frac{\partial u_{1}}{\partial v}\right|^{2} d \Sigma \\
& -\frac{1}{2} \int_{\Sigma_{1}} m_{k} v_{k}\left(a_{2}\left|\nabla u_{2}\right|^{2}-a_{1}\left|\nabla u_{1}\right|^{2}\right) d \Sigma \\
& \left.=\int_{Q}^{\prime}(t), m_{k} \frac{\partial u(t)}{\partial x_{k}}\right)\left.\right|_{0} ^{T}+\frac{n-1}{2} \int_{Q}\left(\left|u^{\prime}\right|^{2}-A(x)|\nabla u|^{2}\right) d x d t \\
& +\int_{0}^{T} E(t) d t-a_{1}\left(1-\frac{a_{1}}{a_{2}}\right) \int_{\Sigma_{1}} m_{k} v_{k}\left|\frac{\partial u_{1}}{\partial v}\right|^{2} d \Sigma \\
& -\frac{1}{2} \int_{\Sigma_{1}} m_{k} v_{k}\left(a_{2}\left|\nabla u_{2}\right|^{2}-a_{1}\left|\nabla u_{1}\right|^{2}\right) d \Sigma .
\end{aligned}
$$

Multiplying (2.1) by $u$ and integrating over $Q$, we obtain

$$
\begin{aligned}
& 0=\left.\left(u^{\prime}, u\right)\right|_{0} ^{T}-\int_{Q}\left|u^{\prime}\right|^{2}-\int_{\Sigma_{1}} a_{1} \frac{\partial u_{1}}{\partial v} u_{1} d \Sigma+\int_{Q_{1}} a_{1}\left|\nabla u_{1}\right|^{2} d x d t \\
& +\int_{\Sigma_{1}} a_{2} \frac{\partial u_{2}}{\partial v} u_{2} d \Sigma+\int_{Q_{2}} a_{2}\left|\nabla u_{2}\right|^{2} d x d t .
\end{aligned}
$$


The transmission conditions give

Therefore, (3.9) becomes

$$
\left.\left(u^{\prime}(t), u(t)\right)\right|_{0} ^{T}=\int_{Q}\left(\left|u^{\prime}\right|^{2}-A(x)|\nabla u|^{2}\right) d x d t .
$$

$$
\begin{aligned}
& \frac{1}{2} \int_{\Sigma} m_{k} v_{k}\left(\left|u_{2}^{\prime}\right|^{2}-a_{2}\left|\nabla_{\sigma} u_{2}\right|^{2}\right) d \Sigma \\
& =\left.\left(u^{\prime}(t), m_{k} \frac{\partial u(t)}{\partial x_{k}}+\frac{n-1}{2} u(t)\right)\right|_{0} ^{T}+T E(0) \\
& -a_{1}\left(1-\frac{a_{1}}{a_{2}}\right) \int_{\Sigma_{1}} m_{k} v_{k}\left|\frac{\partial u_{1}}{\partial v}\right|^{2} d \Sigma \\
& -\frac{1}{2} \int_{\Sigma_{1}} m_{k} v_{k}\left(a_{2}\left|\nabla u_{2}\right|^{2}-a_{1}\left|\nabla u_{1}\right|^{2}\right) d \Sigma
\end{aligned}
$$

To prove (3.8), we have to estimate the right hand of (3.10). First, from the CauchySchwarz's inequality we have

$$
\begin{aligned}
& \left|\left(u^{\prime}(t), m_{k} \frac{\partial u(t)}{\partial x_{k}}+\frac{n-1}{2} u(t)\right)\right| \\
& \leq \frac{R\left(x^{0}\right)}{2 \sqrt{a_{2}}} \int_{\Omega}\left|u^{\prime}(t)\right|^{2} d x+\frac{a_{2}}{2 R\left(x^{0}\right) \sqrt{a_{2}}} \int_{\Omega}\left|m_{k} \frac{\partial u(t)}{\partial x_{k}}+\frac{n-1}{2} u(t)\right|^{2} d x .
\end{aligned}
$$

Moreover,

Since

$$
\begin{aligned}
& \int_{\Omega}\left|m_{k} \frac{\partial u(t)}{\partial x_{k}}+\frac{n-1}{2} u(t)\right|^{2} d x \\
& =\int_{\Omega}\left|m_{k} \frac{\partial u}{\partial x_{k}}\right|^{2} d x+\frac{(n-1)^{2}}{4} \int_{\Omega}|u(t)|^{2} d x+(n-1)\left(m_{k} \frac{\partial u}{\partial x_{k}}, u(t)\right) .
\end{aligned}
$$

$$
\begin{aligned}
\left(m_{k} \frac{\partial u}{\partial x_{k}}, u(t)\right) & =\frac{1}{2} \int_{\Omega} m_{k} \frac{\partial}{\partial x_{k}}\left(|u(t)|^{2} d x\right. \\
& =\frac{1}{2} \int_{\Gamma_{1}} m_{k} v_{k}\left|u_{1}(t)\right|^{2} d \Gamma-\frac{n}{2} \int_{\Omega_{1}}\left|u_{1}(t)\right|^{2} d x \\
& -\frac{1}{2} \int_{\Gamma_{1}} m_{k} v_{k}\left|u_{2}(t)\right|^{2} d \Gamma+\frac{1}{2} \int_{\Gamma} m_{k} v_{k}\left|u_{2}(t)\right|^{2} d \Gamma \\
& -\frac{n}{2} \int_{\Omega_{2}}\left|u_{2}(t)\right|^{2} d x \\
& =\frac{1}{2} \int_{\Gamma} m_{k} v_{k}\left|u_{2}(t)\right|^{2} d \Gamma-\frac{n}{2} \int_{\Omega}|u(t)|^{2} d x,
\end{aligned}
$$


then,

$$
\begin{aligned}
& \int_{\Omega}\left|m_{k} \frac{\partial u(t)}{\partial x_{k}}+\frac{n-1}{2} u(t)\right|^{2} d x \\
& =\int_{\Omega}\left|m_{k} \frac{\partial u(t)}{\partial x_{k}}\right|^{2} d x+\frac{1-n^{2}}{4} \int_{\Omega}|u(t)|^{2} d x \\
& +\frac{n-1}{2} \int_{\Gamma} m_{k} v_{k}\left|u_{2}(t)\right|^{2} d \Gamma \\
& \leq R_{0}^{2} \int_{\Omega}|\nabla u(t)|^{2} d x+\frac{1-n^{2}}{4} \int_{\Omega}|u(t)|^{2} d x \\
& +\frac{n-1}{2} \int_{\Gamma} m_{k} v_{k}|u(t)|^{2} d \Gamma .
\end{aligned}
$$

Thus, (3.11) becomes

$$
\begin{aligned}
& \left|\left(u^{\prime}(t), m_{k} \frac{\partial u(t)}{\partial x_{k}}+\frac{n-1}{2} u(t)\right)\right| \\
& \leq \frac{R\left(x^{0}\right)}{\sqrt{a_{2}}} E(t)+\frac{\sqrt{a_{2}}\left(1-n^{2}\right)}{8 R\left(x^{0}\right)} \int_{\Omega}|u(t)|^{2} d x \\
& +\frac{\sqrt{a_{2}}(n-1)}{4 R\left(x^{0}\right)} \int_{\Gamma} m_{k} v_{k}\left|u_{2}(t)\right|^{2} d \Gamma .
\end{aligned}
$$

Secondly, we estimate the last two terms of (3.10). Since

$$
\left|\nabla u_{1}\right|^{2}=\left|\frac{\partial u_{1}}{\partial v}\right|^{2}+\left|\nabla_{\sigma} u_{1}\right|^{2}, \quad\left|\nabla u_{2}\right|^{2}=\left|\frac{\partial u_{2}}{\partial v}\right|^{2}+\left|\nabla_{\sigma} u_{2}\right|^{2},
$$

and $\nabla_{\sigma} u_{1}=\nabla_{\sigma} u_{2}$ on $\Sigma_{1}$, we deduce that

$$
\begin{aligned}
& -a_{1}\left(1-\frac{a_{1}}{a_{2}}\right) \int_{\Sigma_{1}} m_{k} v_{k}\left|\frac{\partial u_{1}}{\partial v}\right|^{2} d \Sigma-\frac{1}{2} \int_{\Sigma_{1}} m_{k} v_{k}\left(a_{2}\left|\nabla u_{2}\right|^{2}-a_{1}\left|\nabla u_{1}\right|^{2}\right) \\
& =-a_{1}\left(1-\frac{a_{1}}{a_{2}}\right) \int_{\Sigma_{1}} m_{k} v_{k}\left|\frac{\partial u_{1}}{\partial v}\right|^{2} d \Sigma \\
& -\frac{1}{2} \int_{\Sigma_{1}} m_{k} v_{k}\left[\left(\frac{a_{1}^{2}}{a_{2}}-a_{1}\right)\left|\frac{\partial u_{1}}{\partial v}\right|^{2}+\left(a_{2}-a_{1}\right)\left|\nabla_{\sigma} u_{1}\right|^{2}\right] d \Sigma \\
& =\frac{a_{1}\left(a_{1}-a_{2}\right)}{2 a_{2}} \int_{\Sigma_{1}} m_{k} v_{k}\left|\frac{\partial u_{1}}{\partial v}\right|^{2} d \Sigma+\frac{a_{1}-a_{2}}{2} \int_{\Sigma_{1}} m_{k} v_{k}\left|\nabla_{\sigma} u_{1}\right|^{2} d \Sigma \geq 0,
\end{aligned}
$$


since $a_{1} \leq a_{2}$ and $m_{k} v_{\mathrm{k}} \geq 0$ on $\Gamma_{1}$. Therefore, it follows from (3.10), (3.11), and (3.12) that

$$
\begin{aligned}
& \frac{1}{2} \int_{\Sigma} m_{k} v_{k}\left(\left|u_{2}^{\prime}\right|^{2}-a_{2}\left|\nabla_{\sigma} u_{2}\right|^{2}\right) d \Sigma \\
& \geq T E(0)-\frac{2 R\left(x^{0}\right)}{\sqrt{a_{2}}} E(0)-\frac{\sqrt{a_{2}}\left(1-n^{2}\right)}{8 R\left(x^{0}\right)} \int_{\Omega}|u(0)|^{2} d x \\
& -\frac{\sqrt{a_{2}}(n-1)}{4 R\left(x^{0}\right)} \int_{\Gamma} m_{k} v_{k}\left(|u(0)|^{2}+|u(T)|^{2}\right) d \Gamma .
\end{aligned}
$$

This implies (i).

Note that there exists $C>0$ such that

$$
\int_{\Gamma\left(x^{0}\right)}\left(|u(0)|^{2}+|u(T)|^{2}\right) d \Gamma \leq C \int_{\Sigma\left(x^{0}\right)}\left(\left|u^{\prime}\right|^{2}+|u|^{2}\right) d \Sigma .
$$

This is because

and

$$
\begin{aligned}
& \int_{\Gamma\left(x^{0}\right)} T|u(T)|^{2} d \Gamma=\int_{\Gamma\left(x^{0}\right)} \int_{0}^{T} u^{2} d t d \Gamma+\int_{\Gamma\left(x^{0}\right)} \int_{0}^{T} t d u^{2} d \Gamma \\
& \leq(T+1) \int_{\Sigma\left(x^{0}\right)}\left(\left|u^{\prime}\right|^{2}+|u|^{2}\right) d \Sigma,
\end{aligned}
$$

$$
\begin{aligned}
& \int_{\Gamma\left(x^{0}\right)} T|u(0)|^{2} d \Gamma=\int_{\Gamma\left(x^{0}\right)} \int_{0}^{T} u^{2} d t d \Gamma+\int_{\Gamma\left(x^{0}\right)} \int_{0}^{T}(t-T) d u^{2} d \Gamma \\
& \leq(T+1) \int_{\Sigma\left(x^{0}\right)}\left(\left|u^{\prime}\right|^{2}+|u|^{2}\right) d \Sigma .
\end{aligned}
$$

Therefore, Lemma 3.3 gives the following result.

Lemma 3.5. (Inverse inequality) Suppose there exists $x^{0} \in \Omega_{1}$ such that $m(x) \cdot v(x) \geq 0$ on $\Gamma_{1}$, where $v$ is directed towards the exterior of $\Omega_{1}$. Assume $a_{2} \leq a_{1}$ and $T>\frac{2 R\left(x^{0}\right)}{\sqrt{a_{2}}}$. Then for all strong solutions $u$ of (2.1) with initial conditions $\left(u^{0}, u^{1}\right) \in H^{1}(\Omega) \times L^{2}(\Omega)$ and $f=0$, there exists a constant $C(T)>0$ such that

$$
\begin{aligned}
& \int_{\Sigma\left(x^{0}\right)}\left(\left|u_{2}^{\prime}\right|^{2}+\left|u_{2}\right|^{2}\right) d \Sigma+\int_{\Sigma_{*}\left(x^{0}\right)}\left|\nabla_{\sigma} u_{2}\right|^{2} d \Sigma \\
& \geq C(T)\left(\left\|u^{0}\right\|_{H^{1}(\Omega)}^{2}+\left\|u^{\prime}\right\|_{L^{2}(\Omega)}^{2}\right) .
\end{aligned}
$$


4. Main theorem. The main theorem of this paper is as follows.

Theorem 4.1. Suppose there exists $x^{0} \in \Omega_{1}$ such that $m(x) \cdot v(x) \geq 0$ on $\Gamma_{1}$, where $v$ is directed towards the exterior of $\Omega_{1}$. Assume $a_{2} \leq a_{1}$ and $T>\frac{2 R\left(x^{0}\right)}{\sqrt{a_{2}}}$. Then for all initial states

$$
\left\{y^{0}, y^{1}\right\} \in L^{2}(\Omega) \times\left(H^{1}(\Omega)\right)^{\prime},
$$

there exists a control function

$$
g= \begin{cases}g_{0} & \text { on } \Sigma\left(x^{0}\right), \\ g_{1} & \text { on } \Sigma_{*}\left(x^{0}\right),\end{cases}
$$

with $g_{0} \in\left(H^{1}\left(\Sigma\left(x^{0}\right)\right)\right)^{\prime}$ and $g_{1} \in\left(H^{1}\left(\Sigma_{*}\left(x^{0}\right)\right)\right)^{\prime}$ such that the solution $y=y(x, t ; g)$ of (1.1) satisfies (1.2).

Proof. We apply HUM. To do so, we consider the problem:

$$
\left\{\begin{array}{ccc}
u^{\prime \prime}-A(x) \Delta u=0 & \text { in } Q \\
u(x, 0)=u^{0}(x), & u^{\prime}(x, 0)=u^{1}(x) & \text { in } \Omega \\
\frac{\partial u_{2}}{\partial v}=0 & & \text { on } \Sigma \\
u_{1}=u_{2}, & a_{1} \frac{\partial u_{1}}{\partial v}=a_{2} \frac{\partial u_{2}}{\partial v} & \text { on } \Sigma_{1} .
\end{array}\right.
$$

For any $\left\{u^{0}, u^{1}\right\} \in\left(C^{\infty}(\bar{\Omega}) \cap H^{2}\left(\Omega_{1}, \Omega_{2}\right)\right) \times C^{\infty}(\bar{\Omega})$, by Theorem 2.1, problem (4.1) has a unique solution $u$ with

$$
C\left([0, T] ; H^{2}\left(\Omega_{1}, \Omega_{2}\right)\right) \cap C\left([0, T] ; H^{1}(\Omega)\right) .
$$

Define

$$
\left\|\left\{u^{0}, u^{1}\right\}\right\|_{F}=\left(\int_{\Sigma\left(x^{0}\right)}\left(\left|u_{2}^{\prime}\right|^{2}+\left|u_{2}\right|^{2}\right) d \Sigma+\int_{\Sigma_{*}\left(x^{0}\right)}\left|\nabla_{\sigma} u_{2}\right|^{2} d \Sigma\right)^{1 / 2},
$$

which is a norm on $\left(C^{\infty}(\bar{\Omega}) \cap H^{2}\left(\Omega_{1}, \Omega_{2}\right)\right) \times C^{\infty}(\bar{\Omega})$, due to Lemma 3.5. Let $F$ be the completion of $\left(C^{\infty}(\bar{\Omega}) \cap H^{2}\left(\Omega_{1}, \Omega_{2}\right)\right) \times C^{\infty}(\bar{\Omega})$ with respect to the norm $\|\cdot\|_{F}$. Then Lemma 3.5 implies that

$$
F \subset H^{1}(\Omega) \times L^{2}(\Omega)
$$

consequently

$$
\left(H^{1}(\Omega)\right)^{\prime} \times L^{2}(\Omega) \subset F^{\prime}
$$


According to the definition of $F$, we have for any $\left\{u^{0}, u^{1}\right\} \in F$,

$$
\left.u\right|_{\Sigma\left(x^{0}\right)},\left.\quad u^{\prime}\right|_{\Sigma\left(x^{0}\right)} \in L^{2}\left(\Sigma\left(x^{0}\right)\right),\left.\quad \nabla_{\sigma} u\right|_{\Sigma_{*}\left(x^{0}\right)} \in\left(L^{2}\left(\Sigma_{*}\left(x^{0}\right)\right)\right)^{n} .
$$

To apply the HUM, we need to consider the backward problem:

$$
\left\{\begin{array}{cc}
\phi^{\prime \prime}-A(x) \Delta \phi=0 & \text { in } Q, \\
\phi(T)=\phi^{\prime}(T)=0 & \text { in } \Omega, \\
\phi_{1}=\phi_{2}, \quad a_{1} \frac{\partial \phi_{1}}{\partial v}=a_{2} \frac{\partial \phi_{2}}{\partial v} & \text { on } \Sigma_{1}, \\
\frac{\partial \phi}{\partial v}=\left\{\begin{array}{cc}
-u_{2}+\frac{\partial}{\partial t} u_{2}^{\prime} & \text { on } \Sigma\left(x^{0}\right), \\
\Delta_{\Gamma_{*}\left(x^{0}\right) u_{2}} & \text { on } \Sigma_{*}\left(x^{0}\right) .
\end{array}\right.
\end{array}\right.
$$

For the definition of the operator $\Delta_{\Gamma_{*}}\left(x^{0}\right)$, see [6, p.138]. The solution of (4.2) can be defined by the transposition method (see [8]) as follows.

Definition 4.2. $\phi$ is said to be a weak solution of (4.2) if there exist $\left\{\rho^{1},-\rho^{0}\right\} \in F^{\prime}$ such that $\phi$ satisfies

$$
\begin{aligned}
& \int_{Q} f \phi d x d t-\left(\rho^{0}, \theta^{1}\right)+\left(\rho^{1}, \rho^{0}\right) \\
& =\int_{\Sigma\left(x^{0}\right)}\left(\theta_{2} u_{2}+\theta_{2}^{\prime} u_{2}^{\prime}\right) d \Sigma+\int_{\Sigma_{*}\left(x^{0}\right)} a_{2} \nabla_{\sigma} \theta_{2} \nabla_{\sigma} u_{2} d \Sigma,
\end{aligned}
$$

for any $\left\{\theta^{0}, \theta^{1}\right\} \in F, f \in L^{1}\left(0, T ; H^{1}(\Omega, 0)\right)$, and where $\theta$ is the solution of the following problem:

$$
\left\{\begin{array}{cc}
\theta^{\prime \prime}-A(x) \Delta \theta=f & \text { in } Q \\
\theta(0)=\theta^{0}, \theta^{\prime}(0)=\theta^{1} & \text { in } \Omega \\
\frac{\partial \theta_{2}}{\partial v}=0 & \text { on } \Sigma \\
\theta_{1}=\theta_{2}, \quad a_{1} \frac{\partial \theta_{1}}{\partial v}=a_{2} \frac{\partial \theta_{2}}{\partial v} & \text { on } \Sigma_{1}
\end{array}\right.
$$

We define $\phi(0)=\rho^{0}, \phi^{\prime}(0)=\rho^{1}$.

Lemma 4.3. Problem (4.2) has a unique solution in the sense of Definition 4.2 satisfying

$$
\begin{gathered}
\phi \in L^{\infty}\left(0, T ;\left(H^{1}(\Omega, 0)\right)^{\prime}\right), \\
\left\{\phi^{\prime}(0),-\phi(0)\right\} \in F^{\prime} .
\end{gathered}
$$


Moreover, there exists $C>0$ such that

$$
\left\|\left\{\phi^{\prime}(0),-\phi(0)\right\}\right\|_{F^{\prime}} \leq C\left\|\left\{u^{0}, u^{1}\right\}\right\|_{F} .
$$

We admit this lemma for the moment. We now define a linear operator $\Lambda$ by

$$
\Lambda\left\{u^{0}, u^{1}\right\}=\left\{\phi^{\prime}(0),-\phi(0)\right\}
$$

Taking $f=0$ in (4.3), we find

$$
\begin{aligned}
& \left\langle\Lambda\left\{u^{0}, u^{1}\right\},\left\{u^{0}, u^{1}\right\}\right\rangle \\
& =\left(\phi^{\prime}(0), u^{0}\right)-\left(\phi(0), u^{1}\right) \\
& =\int_{\Sigma\left(x^{0}\right)}\left(\left|u_{2}^{\prime}\right|^{2}+\left|u_{2}\right|^{2}\right) d \Sigma+\int_{\Sigma_{*}\left(x^{0}\right)} a_{2}\left|\nabla_{\sigma} u_{2}\right|^{2} d \Sigma .
\end{aligned}
$$

Lemma 3.5, Lemma, 4.3, and the Lax-Milgram Theorem show that $\Lambda$ is an isomorphism from $F$ to $F^{\prime}$. This means that for all $\left\{\mathrm{y}^{1},-\mathrm{y}^{0}\right\} \in F^{\prime}$, the equation

$$
\Lambda\left\{u^{0}, u^{1}\right\}=\left\{y^{1},-y^{0}\right\}
$$

has a unique solution $\left\{u^{0}, u^{1}\right\}$. With this initial condition we solve problem (4.1), and then solve problem (4.2). Then we have found the control function

$$
g=\left\{\begin{array}{cc}
-u_{2}+\frac{\partial}{\partial t} u_{2}^{\prime}, & \text { on } \Sigma\left(x^{0}\right) \\
\Delta_{\Gamma_{*}\left(x^{0}\right)} u_{2}, & \text { on } \Sigma_{*}\left(x^{0}\right) .
\end{array}\right.
$$

with $g_{0}=-u_{2}+\frac{\partial}{\partial \tau} u_{2}^{\prime} \in\left(H^{1}\left(\Sigma\left(x^{0}\right)\right)\right)^{\prime} \quad$ and $\quad g_{1}=\Delta_{\Gamma_{*}\left(x^{0}\right)} u_{2} \in\left(H^{1}\left(\Sigma_{*}\left(x^{0}\right)\right)\right)^{\prime} \quad$ such that

$$
y(x, t ; g)=\phi(x, t ; g)
$$

is the solution of (1.1) satisfying (1.2). Thus, we have proved Theorem 4.1 provided we can prove Lemma 4.3.

Proof of Lemma 4.3. The solution $\theta$ of problem (4.4) can be written as $\theta=v+w$, where $v$ and $w$ are, respectively, solutions of the following problems:

$$
\left\{\begin{array}{cc}
v^{\prime \prime}-A(x) \Delta v=0 & \text { in } Q, \\
v(x, 0)=\theta^{0}(x), v^{\prime}(x, 0)=\theta^{1}(x) & \text { in } \Omega, \\
\frac{\partial v_{2}}{\partial v}=0 & \text { on } \Sigma, \\
v_{1}=v_{2}, \quad a_{1} \frac{\partial v_{1}}{\partial v}=a_{2} \frac{\partial v_{2}}{\partial v} & \text { on } \Sigma_{1},
\end{array}\right.
$$


and

$$
\left\{\begin{array}{cc}
w^{\prime \prime}-A(x) \Delta w=f & \text { in } Q \\
w(x, 0)=u^{\prime}(x, 0)=0 & \text { in } \Omega \\
\frac{\partial w_{2}}{\partial v}=0 & \text { on } \Sigma \\
w_{1}=w_{2}, \quad a_{1} \frac{\partial w_{1}}{\partial v}=a_{2} \frac{\partial w_{2}}{\partial v} & \text { on } \Sigma_{1}
\end{array}\right.
$$

Since $\left\{\theta^{0}, \theta^{1}\right\} \in F$, we have

$$
\left\|\left\{\theta^{0}, \theta^{1}\right\}\right\|_{F}=\left(\int_{\Sigma\left(x^{0}\right)}\left(\left|v_{2}^{\prime}\right|^{2}+\left|v_{2}\right|^{2}\right) d \Sigma+\int_{\Sigma_{*}\left(x^{0}\right)}\left|\nabla_{\sigma} v_{2}\right|^{2} d \Sigma .\right.
$$

On the other hand, by Theorem 2.1 and the trace theorem (see [7, Chapter 1]), we have

$$
\left(\int_{\Sigma\left(x^{0}\right)}\left(\left|w_{2}^{\prime}\right|^{2}+\left|w_{2}\right|^{2}\right) d \Sigma+\int_{\Sigma_{*}\left(x^{0}\right)}\left|\nabla_{\sigma} w_{2}\right|^{2} d \Sigma\right)^{1 / 2} \leq C\|f\|_{L^{1}\left(0, T ; H^{1}(\Omega)\right)} .
$$

Therefore,

$$
\begin{aligned}
& \left|\int_{Q} f \phi d x d t-\left(\rho^{0}, \theta^{1}\right)+\left(\rho^{1}, \theta^{0}\right)\right| \\
& =\left|\int_{\Sigma\left(x^{0}\right)}\left(\theta_{2} u_{2}+\theta_{2}^{\prime} u_{2}^{\prime}\right) d \Sigma+\int_{\Sigma_{*}\left(x^{0}\right)} a_{2} \nabla_{\sigma} \theta_{2} \nabla_{\sigma} u_{2} d \Sigma\right| \\
& \leq\left|\int_{\Sigma\left(x^{0}\right)}\left(v_{2} u_{2}+v_{2}^{\prime} u_{2}^{\prime}\right) d \Sigma+\int_{\Sigma_{*}\left(x^{0}\right)} a_{2} \nabla_{\sigma} v_{2} \nabla_{\sigma} u_{2} d \Sigma\right| \\
& +\left|\int_{\Sigma\left(x^{0}\right)}\left(w_{2} u_{2}+w_{2}^{\prime} u_{2}^{\prime}\right) d \Sigma+\int_{\Sigma_{*}\left(x^{0}\right)} a_{2} \nabla_{\sigma} w_{2} \nabla_{\sigma} u_{2} d \Sigma\right| \\
& \leq C\left(\left\|\left\{\theta^{0}, \theta^{1}\right\}\right\|_{F}+\|f\|_{L^{1}\left(0, T ; H^{1}(\Omega)\right)}\right)\left\|\left\{u^{0}, u^{1}\right\}\right\|_{F} .
\end{aligned}
$$

Thus, there exist $\phi \in L^{\infty}\left(0, T ;\left(H^{1}(\Omega, 0)\right)^{\prime}\right)$ and $\left\{\rho^{0},-\rho^{1}\right\} \in F^{\prime}$ such that (4.3) holds. That is, $\phi$ is a weak solution of (4.2) and $\left\{\phi(0),-\phi^{\prime}(0)\right\} \in F^{\prime}$. Taking $f=0,(4.10)$ gives (4.5).

REMARK 4.4. If $\Omega$ is star-shaped with respect to $x^{0}$, then $\Sigma_{*}\left(x^{0}\right)=\phi$. In this case, we obtain a control function $g$ with $g \in\left(H^{1}\left(0, T ; L^{2}(\Gamma)\right)\right)^{\prime}$.

Acknowledgements. The authors thank the referees for their helpful comments which have made the original version more concise. The first author is grateful for the Overseas Postgraduate Research Scholarship from Australian 
Government, the University of Wollongong Postgraduate Research Award, and the Department of Mathematics Analysis Research Group Scholarship from the Faculty of Informatics at the University of Wollongong.

\section{REFERENCES}

1. G. Chen, Energy decay estimates and exact boundary value controllability for the wave equation in a bounded domain, J. Math. Pures Appl. 58 (1979), 249-273.

2. G. A. Chen, A note on the boundary stabilization of the wave equation, SIAM J. Control Optim 19 (1981), 106-113.

3. R. Dautray and J. L. Lions, Mathematical analysis and numerical methods for science and technology, Vol. 2, Functional and variational methods; Vol. 5, Evolution problems I (Springer-Verlag, 1992).

4. P. Grisvard, Contrôlabilité Exacte des Solutions de L'équation des Ondes en Présence de Singularités, J. Math. Pures Appl. 68 (1989), 215-259.

5. J. K. Hale, Ordinary differential equations (Wiley-Interscience, 1969).

6. J. L. Lions, Contrôlabilité Exacte Perturbations et Stabilisation de Systèmes Distribuées, Tome1, Contrôlabilité Exacte (Masson, 1988).

7. J. L. Lions, Exact controllability, stabilization and perturbations for distributed systems, SIAM Rev. 30 (1988), 1-68.

8. J. L. Lions and E. Magenes, Non-homogeneous boundary value problems and applications, Vol. I and II (Springer-Verlag, 1972).

9. S. Nicaise, Exact controllability of a pluridimensional coupled problem, Rev. Mat. Univ. Complut. Madrid 5 (1992), 91-135.

10. S. Nicaise, Contrôlabilité exacte frontière des probléme de transmission avec singularites, C. R. Acad. Sci., Paris, Séres I, Math. 320 (1995), 663-668.

11. S. Nicaise, Contrôlabilité exacte frontière des problèm de transmission par adjonction de contrôles internees, C. R. Acad. Sci., Paris. Séres I. Math. 321 (1995), 969-974.

12. S. Nicaise, Boundary exact controllability of interface problems with singularities: addition of the coefficients singularities, SIAM J. Control Optim. 34 (1996), 1512-1532

13. D. L. Russell, Controllability and stabilizability theory for linear partial differential equations: recent progress and open questions, SIAM Rev. 20 (1978), 639-739.

14. D. L. Russell, Exact boundary value controlability theorems for wave and heat processes in star-complemented regions, in Differential Games and Control Theory, Roxin, Liu, and Sternberg, Eds., (Marcel Dekker Inc., 1974), 291-319. 